\title{
Alterations of the treatment-naive gut microbiome in newly diagnosed Hepatitis C Virus infection
}

\author{
Salma Sultan ${ }^{5, \$}$, Mohammed El-Mowafy, \$, Abdelaziz Elgaml 1,2, \$, Mohamed El-Mesery ${ }^{3}$, \\ Ahmed El Shabrawi ${ }^{4}$, Mohamed Elegezy ${ }^{4}$, Riadh Hammami ${ }^{* *}$ and Walid Mottawea ${ }^{1,5^{*}}$ \\ ${ }^{1}$ Faculty of Pharmacy, Department of Microbiology and Immunology, Mansoura University, \\ Mansoura 35516, Egypt. \\ ${ }^{2}$ Faculty of Pharmacy, Department of Microbiology and Immunology, Horus University, New \\ Damietta 34518, Egypt. \\ ${ }^{3}$ Faculty of Pharmacy, Department of Biochemistry, Mansoura University, Mansoura 35516, \\ Egypt. \\ ${ }^{4}$ Faculty of Medicine, Department of Endemic Hepatology and Gastroenterology, and \\ Department of Tropical Medicine, Mansoura University, Mansoura 35516, Egypt. \\ ${ }^{5}$ Faculty of Health Sciences, School of Nutrition Sciences, University of Ottawa, Ottawa, Ontario \\ K1H8M5, Canada. \\ \$ These authors contributed equally to this work. \\ * Correspondence: \\ Walid Mottawea \\ wmott020@uottawa.ca and \\ Riadh Hammami \\ riadh.hammami@uottawa.ca
}


a)

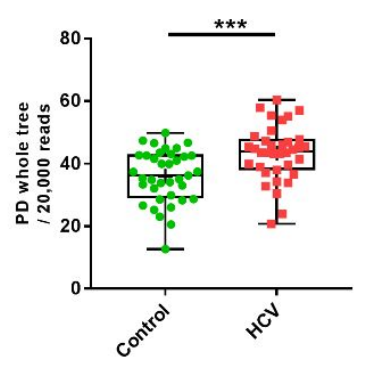

b)

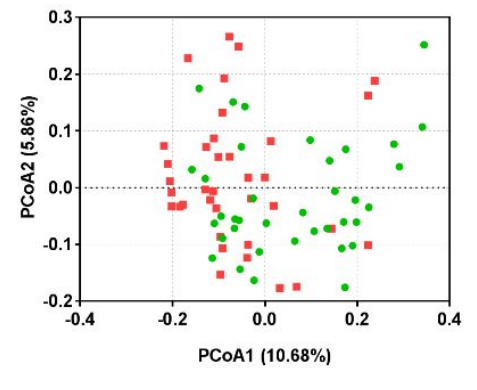

c)

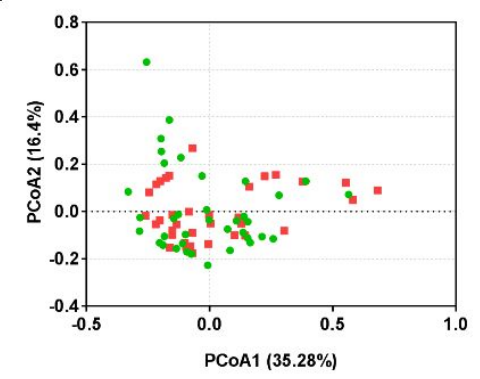

- $\mathrm{HCV} \quad$ Control

Figure S1: Gut microbiota from HCV patients is more diverse compared to healthy subjects based on phylogenetic measures. a) PD whole tree indices of the gut microbiota from healthy subjects and HCV patients calculated by QIIME 1.9.1 using rarified 20,000 reads from each sample. Mann-Whitney two tailed test was used for statistical analysis. $* * * P=0.001$. b) and c) Principal Coordinate Analyses (PCoA) based on Unweighted UniFrac (b) and weighted UniFrac (c) distances. UniFrac distances were calculated and PCoA was conducted using QIIME 1.9.1 and randomly selected 20,000 reads per sample. Samples are colored by disease status. PCoA1 and PCoA2 represent the top two coordinates with the highest variability among samples and the percentage shown indicates the fraction of variation captured by each coordinate. 


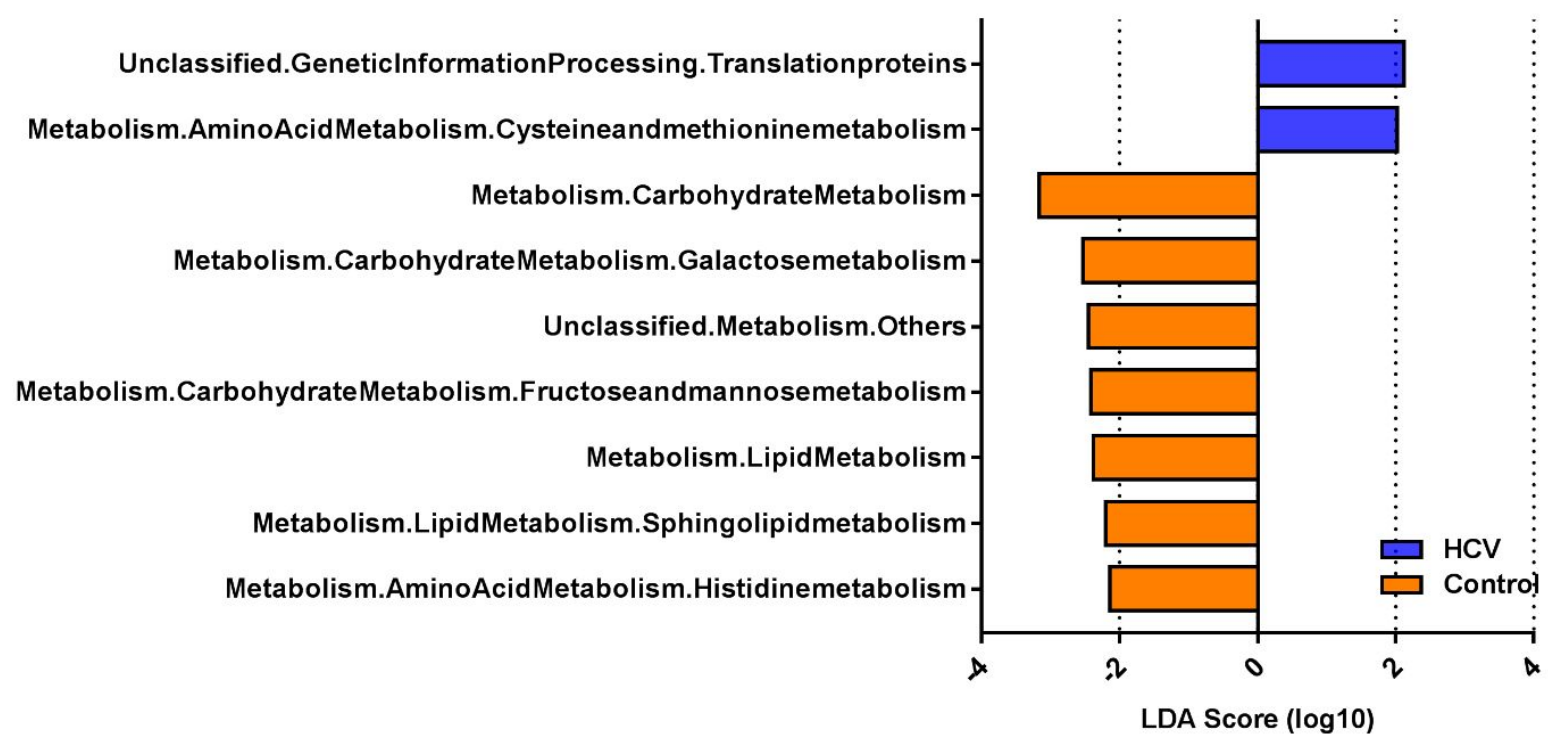

Figure S2: Differences in gut microbiota functionalities in HCV infection. A histogram of linear discriminant analysis (LDA) effect size score for HCV differentially abundant KEGG orthologs compared with controls $(\mathrm{n}=38$ each); the presented functions exhibit an LDA significant threshold $\geq 2$ and a $\mathrm{P}<0.05$ using a pairwise Wilcoxon test. OTU table with cumulative sum scaling normalized counts was used to predict community metagenomics and KEGG Orthologs through Phylogenetic Investigation of Communities by Reconstruction of Unobserved States (PICRUSt v1.1.4). The generated categorized functions were then analyzed by LDA Effect Size (LEfSe 1.1) (Segata et. Al, 2011). 
ROC curve: (g) Bacteroides (OTU_348304)

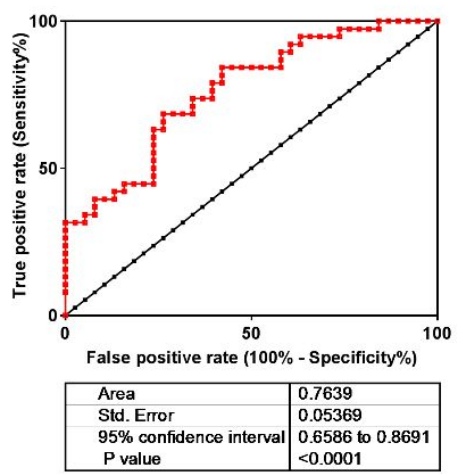

ROC curve: (g) Megas phaera (OTU_266210)

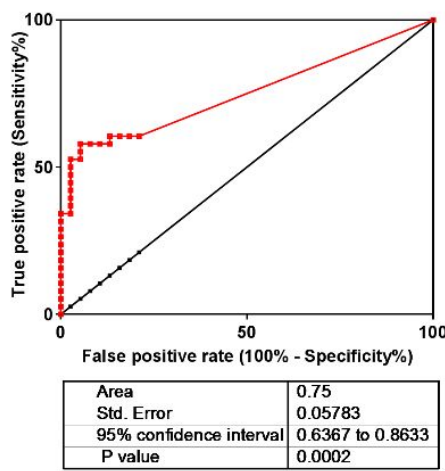

ROC curve: (s) Prevotella stercorea (OTU_522959)

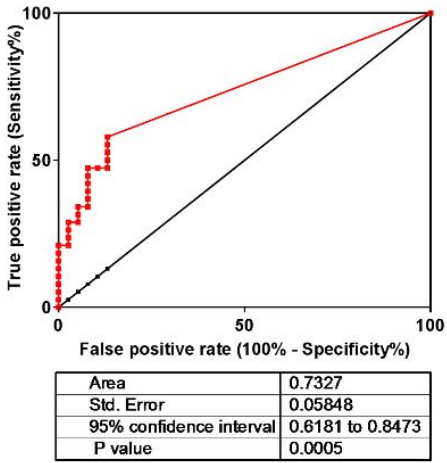

ROC curve: (g) Succinivibrio (OTU_591731)

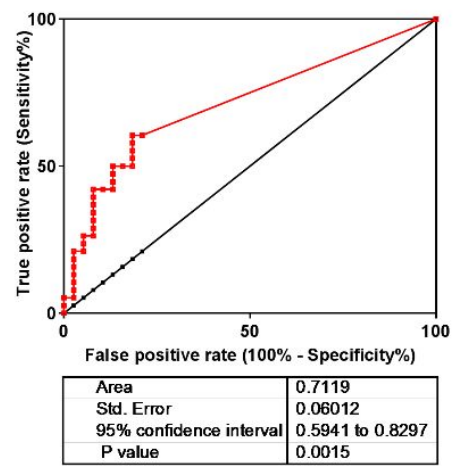

ROC curve: (g) Streptococcus (OTU_593803)

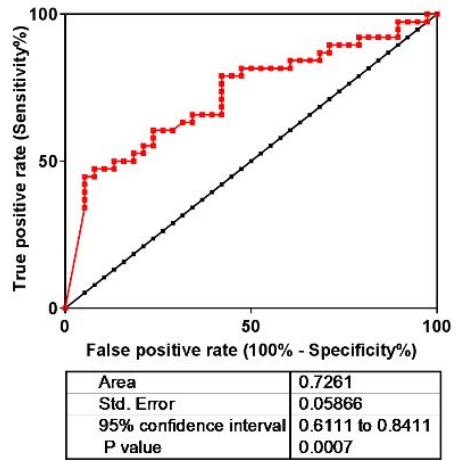

- Sensitivity\%

- Identity $\%$

Figure S3: Potential microbiota biomarkers of HCV infection. Receiver operating characteristic (ROC) curve for differentiation of $\mathrm{HCV}$ infected patients from healthy subjects and the associated statistics based on the normalized read count of the indicated OTUs. 


\section{References}

Segata, N., Izard, J., Waldron, L., Gevers, D., Miropolsky, L., Garrett, W.S., and Huttenhower, C., Metagenomic biomarker discovery and explanation. Genome Biol, 2011. 12: R60. 